Resumen por el autor, Lewis V. Heilbrunn.

Cambios de la viscosidad del protoplasma durante la mitosis.

En estudios precedentes el autor ha observado los cambios de la viscosidad del protoplasma durante la mitosis. En el presente trabajo ha intentado determinar la magnitud de estos cambios y su exacta relación temporal con el proceso de la mitosis. El metodo de la centrifugacion ha sido empleado para medir la viscosidad del protoplasma. La mayor parte de las medidas fueron llevadas a cabo en el ovulo del lamelibranquio Cumingia. Los ovulos fueron centrifugados con cortos intervalos durante el tiempo transcurrido entre la fecundacion y segmentacion. La velocidad del movimiento granular bajo la influencia de una fuerza centrifuga determinada han sido considerada como la medida de la viscosidad protoplasmica. Tanto durante las divisiones de maduracion como en la segmentacion, la aparición del huso esta precedida por una aumento brusco en la viscosidad, seguido de una brusca disminucion de la misma. Al final de la mitosis, inmediatamente antes de dividirse la celula, la viscosidad aumenta marcadamente. Las medidas cuantitativas han sido obtenidas y los resultados han sido representados graficamente. Durante las divisiones de maduración y la segmentacion del ovulo del anelido Nereis tienen lugar cambios de viscosidad semejantes a los de Cumingia.

Translation by José F. Nonidez Cornell Medical College, New York 
AUTROR'S ABSTRACT OF THIS PAPER ISSUED BY THE RIBLIOGRAPHIC SERVICE, NOVEMBER 7

\title{
PROTOPLASMIC VISCOSITY CHANGES DURING MITOSIS ${ }^{1}$
}

\author{
L. V. HEILBRUNN
}

ONE CHART

THE PROBLEM

Earlier work showed that the segmentation of the sea-urchin egg and the appearance of the mitotic spindle in this egg depend upon a viscosity increase in the cytoplasm (Heilbrunn," 15, '17, '20). During mitosis protoplasmic viscosity changes were demonstrated. It is of interest now to inquire whether similar viscosity shanges occur in the dividing eggs of other forms. If the mitotic mechanism depends upon viscosity changes, then such changes should be present in all dividing cells. Furthermore, it has been thought advisable to determine more accurately the magnitude of these changes.

Most of the work was done with the Cumingia egg, although some data from the sea-urchin and Nereis eggs is included for the sake of comparison. The Cumingia egg is immature at fertilization and therefore offers an interesting comparison to the egg of the sea-urchin. In Cumingia, three cell divisions occur in a short space of time between fertilization and the first cleavage. Do viscosity changes accompany these divisions and do they show the same time relation to the mitotic process that was previously demonstrated for the sea-urchin egg?

At Woods Hole Cumingia tellinoides is fairly common, and during the summer of 1920 good material was generally available throughout July and August. My thanks are due to Prof. F. R. Lillie, director of the Marine Biological Laboratory, for the priviuge of carrying on the work there.

1 Contribution from the Zoological Laboratory of the University of Michigan. 


\section{THE METHOD}

In general the viscosity of a fluid may be determined in any one of three ways. The usual method is to measure the rate of flow of the fluid through a tube. One can also obtain a measure of the viscosity by determining the rate at which solid substances pass through the fluid, or one can measure viscosity by determining the force necessary to push solid materials through the fluid. All three methods are used by physicists, but only the second and third can be applied to the living cell. When the protoplasm is not very viscous and contains large granules of relatively high specific gravity, these granules may be seen to move under the influence of gravity. The time of fall can then be used as a measure of the viscosity of the protoplasm. This method has been applied to some plant cells by Heilbronn ('14). In animal cells generally the granules do not appear to be affected by gravity, and a stronger force is necessary to move them. Such a force can be obtained with the centrifuge, and the centrifugal method of measuring viscosity is doubtless capable of wide application. With the centrifuge both the second and third methods of measuring viscosity can be used. One can either determine the speed attained by protoplasmic granules under the influence of a given centrifugal force or one can determine the amount of force necessary to produce a certain degree of movement. To some extent both methods were used in earlier work.

At this point it might be well to consider the theory of viscosity measurements obtained by observing the movements of solids through liquids. This method has been used comparatively rarely by physicists and chemists. Arndt ('07) used it to determine the viscosity of fused salts. A general discussion of the subject with references to literature is given by Ladenburg ('07). When fairly small particles are made to move through viscous liquids, this movement is found to occur at a uniform rate. For such a movement in the case of spherical particles, Stokes ('50) derived a formula often used by physicists. In a suitable form it is: 


$$
V=\frac{2 g(\sigma-\rho) a^{2}}{9 \mu}
$$

in which $\mathrm{V}$ is the velocity of movement, $g$ the gravity constant, $\sigma$ the specific gravity of the particle, $\rho$ the specific gravity of the liquid, $a$ the radius of the particle, and $\mu$ the viscosity of the liquid.

In the mathematical derivation of Stokes' formula various assumptions are made. Arnold ('11) has considered the significance of all these assumptions. He has shown that for small particles dropping through viscous liquids, Stokes' law holds. When the particles drop through comparatively narrow tubes of the liquid, then a correction must be made for the effect of the walls of the tube. For comparative tests of viscosity in which the same tube is used throughout, this correction disappears.

Stokes' law applies to the action of gravity. In order to make it apply to centrifugal force, one must insert a factor $c$ for the centrifugal force in terms of gravity. The formula then becomes:

$$
V=\frac{2 c g(\sigma-\rho) a^{2}}{9 \mu}
$$

Centrifugal force may be many times gravity. It might be thought that with such a powerful force, the velocity of the particles might become so great that Stokes' law would no longer hold. In the present experiments no such high velocity was ever attained. The speed of the granules ranged approximately from 0.0002 to $0.0018 \mathrm{~cm}$. per second. This is a very low rate of speed.

We are reasonably safe, therefore, in assuming that Stokes' law can be applied to the movement of cytoplasmic granules under the influence of centrifugal force. With a given centrifugal force, the speed with which the granules move is accordingly inversely proportional to the viscosity of the fluid through which they move, the cytoplasm. But this speed also depends on two other factors, the radius of the particle, and the difference in specific gravity between the particle and the fluid of the cytoplasm. If the simple formula is to be used to measure viscosity 
it is important that these factors remain constant. During mitosis in the Cumingia egg the granules do not change appreciably in size. This constancy is shown in the drawings of Morgan ('10). ${ }^{2}$ Perhaps the specific gravity of the granules changes. In the egg there are two sets of granules, one set lighter, the other heavier than the cytoplasm. When the eggs are centrifuged, the lighter granules go to one pole, the heavier granules to the opposite pole. It has been found that whenever the lighter granules move more rapidly, the heavier granules also move more rapidly, and a drop in the speed of the lighter granules is always accompanied by a drop in the speed of the heavier granules. If the change in speed were in any case due to a change in specific gravity of the granules, we would have to assume that an increase in the specific gravity of the lighter granules was in every case accompanied by a corresponding decrease in the specific gravity of the heavier granules; and vice versa. At first sight this appears impossible, but it is not. An entrance of water into the granules would increase the specific gravity of the lighter granules and decrease the specific gravity of the heavier granules, and an exit of water would have the opposite effect. But such a passage of water would of necessity produce very decided changes in the diameter of the granules and these changes, as we have already noted, do not occur.

Since, therefore, the diameter of the granules and their specific gravity can be taken as constant, the velocity of the granules affords a measure of the viscosity of the cytoplasm. In measuring changes in protoplasmic viscosity, one can either determine the variation in the speed of the granules with a certain amount of centrifugal force or one can determine the amount of force necessary to produce a certain velocity of the granules. In actual practice it is easier to use the first method, especially if a hand centrifuge is employed. With such a centrifuge one acquires the knack of turning very regularly at a given speed but it is somewhat more difficult to change from one speed to another.

2 It was also shown by careful measurement of granules in living eggs. 
In all of the measurements made in 1920 a new model Bausch \& Lomb centrifuge with haematocrit attachment was used. With this instrument one turn of the high-speed handle produces 130 turns of the tubes. The distance between the ends of the tubes is about $14.6 \mathrm{~cm}$. The centrifugal force in dynes is given by the formula of Huyghens:

$$
C=4 \pi^{2} n^{2} r m .
$$

in which $C$ is the centrifugal force, $n$ is the number of turns per second, $r$ is the radius of the circle described by the ends of the tubes, and $m$ the mass. The force of gravity is 980.36 dynes (at Boston) times $m$. In order to express the centrifugal force in terms of gravity, we must therefore divide by $980.36 \mathrm{~m}$. Thus:

$$
C^{\prime}=\frac{4 \pi^{2} n^{2} r m}{g m}=\frac{4 \pi^{2} n^{2} r}{g}
$$

in which $C^{\prime}$ is the centrifugal force in terms of gravity, and $g$ is the gravity constant. With the hand centrifuge it was found most convenient to maintain a speed of one turn of the highspeed handle per second. This represents a force 4968 times gravity.

When the Cumingia egg is centrifuged, the oil globules pass to one pole, the yolk and pigment granules to the opposite pole. The egg then appears banded and three zones show, a dark zone contains the oil globules, a pigmented zone contains the yolk granules and the pink pigment, and between the two is a clear and transparent zone. In referring to these zones they will be spoken of as the dark zone, the pigment zone, and the hyaline zone. The appearance of these zones is beautifully shown in the illustrations of Morgan.

\section{THE VISCOSITY CHANGES IN THE CUMINGIA EGG}

The protoplasm of the unfertilized Cumingia egg has a relatively low viscosity. A number of tests were made during the season. At a speed of one turn per second of the high-speed 
handle of the centrifuge, usually two or three seconds elapsed before the first indication of zones appearea. In one case four seconds were required, but this was exceptional. In another case a single turn was sufficient to show zones, but these eggs of unusually low viscosity were not absolutely normal and at segmentation they showed poorly indented cleavage furrows. Apparently the viscosity varies with the temperature, decreasing slightly as the temperature rises from $19^{\circ}$ to $25^{\circ}$. More data are necessary, however, in order to establish this point.

The viscosity changes during mitosis are shown in the following tables. Eggs were centrifuged at rapid intervals between fertilization and cleavage. One series of tests was made in which the centrifuge handle was turned three times in three seconds, another series in which it was turned four times in four seconds, and so on. In all of the tests recorded here, a uniform speed of one turn per second was maintained. This was continued for from two to twenty seconds in the various tests. Because of the difficulty in starting, it is not easy to turn the handle twice in exactly two seconds. After a turn or two has been made, however, it is a simple matter to keep the speed uniform.

In the tables the first column shows the number of seconds elapsed after fertilization at the time a given test was made. In working with various sets of eggs on different days, the length of time between fertilization and cleavage varies considerably. In order to compare one set of eggs with another, it is necessary to reduce all the time records to some standard basis. The average time between fertilization and cleavage is about fifty minutes. Fifty minutes was therefore taken as a standard time interval, and by a simple calculation it was determined in each case how much time would have elapsed if the total time until cleavage had been fifty minutes. These figures are given in the second column and are referred to as 'standard time.' There is always some variation in the time of cleavage of individual eggs of any one batch. The time of first cleavage was taken as the time when 50 per cent of the eggs had completely divided. In order to obtain this time, rapid counts had to be made at frequent intervals. These counts are recorded in the third 
column in the form of fractions. The numerator represents the number of eggs completely segmented, the denominator the total number of eggs counted.

In the third column also are recorded the observations made during the progress of the tests. It must be pointed out that these observations were necessarily very hasty, and are therefore not as accurate as they might have been if the observer had had time to proceed leisurely. Usually the tests were made at very frequent intervals, and all observations had to be completed in the shortest possible time.

TABLE 1

Three-second tests. Temperature, $24^{\circ}$

\begin{tabular}{|c|c|c|}
\hline $\begin{array}{l}\text { AGHUALTMME } \\
\text { AFTER } \\
\text { FERTILIZATION }\end{array}$ & $\begin{array}{l}\text { ETANDARD } \\
\text { TIME }\end{array}$ & OBSERVATIONS \\
\hline minutes & minutes & \\
\hline 5 & 4.9 & Dark zone distinct in some eggs \\
\hline $7 \frac{1}{2}$ & 7.3 & $\begin{array}{l}3 \text { zones appear in some eggs; others lack zones, these } \\
\text { have polar body. }\end{array}$ \\
\hline 10 & 9.7 & No zones visible. All eggs have polar body. \\
\hline $12 \frac{1}{2}$ & 12.1 & No zones. \\
\hline 15 & 14.5 & No zones. \\
\hline $17 \frac{1}{2}$ & 17.0 & $\begin{array}{l}\text { A few eggs show zones (perhaps } 10 \text { to } 20 \text { per cent). } \\
\text { Most eggs lack zones. }\end{array}$ \\
\hline 20 & 19.4 & Dark zone present, often it is not very sharply defined. \\
\hline $22 \frac{1}{2}$ & 21.8 & No zones. \\
\hline 25 & 24.3 & No zones. \\
\hline $27 \frac{1}{2}$ & 26.7 & No zones. \\
\hline 30 & 29.1 & $\begin{array}{l}\text { No zones. The two pronuclei appear large, but they } \\
\text { are not yet apposed. }\end{array}$ \\
\hline $32 \frac{1}{2}$ & 31.6 & No zones. \\
\hline 35 & 34.0 & No zones. The pronuclei are apposed. \\
\hline $37 \frac{1}{2}$ & 36.4 & No zones. The pronuclei have fused. \\
\hline 40 & 38.8 & $\begin{array}{l}\text { Most eggs lack zones. A few show the dark zone, but } \\
\text { it is not very sharply marked off. }\end{array}$ \\
\hline 43 & 41.7 & $\begin{array}{l}\text { Eggs generally lack zones. } 1 \text { or } 2 \text { perhaps show dark } \\
\text { zone. }\end{array}$ \\
\hline 46 & 44.7 & No zones. \\
\hline $48 \frac{1}{2}$ & 47.1 & $\begin{array}{l}\text { No zones. Many eggs in mitotic elongation stage. } \\
\text { Some are beginning to segment. }\end{array}$ \\
\hline $50-51$ & & $12 / 100$ segmented. \\
\hline $51 \frac{1}{2}$ & & $\begin{array}{l}23 / 50 \text { segmented. This taken as the time of segmen- } \\
\text { tation. }\end{array}$ \\
\hline $52 \frac{1}{2}$ & & $37 / 50$ segmented. \\
\hline
\end{tabular}


TABLE 2

Four-second tests. Eggs fertilized at 4.30 p.m. Temperature at 4.29 p.m., $25.6^{\circ}$; at 5.15 p.m., $25.8^{\circ}$

\begin{tabular}{|c|c|c|}
\hline $\begin{array}{c}\text { ACTUAL TIME } \\
\text { AFTER } \\
\text { FERTILAATION }\end{array}$ & $\begin{array}{l}\text { BTANDARD } \\
\text { TIME }\end{array}$ & OBSERVATTONS \\
\hline minutes & minutes & \\
\hline 2 & 2.4 & Three zones show plainly. \\
\hline 4 & 4.8 & $\begin{array}{l}\text { In some eggs zones appear faintly. Most eggs lack } \\
\text { zones. A few polar bodies observed (5 min. after } \\
\text { fertilization). }\end{array}$ \\
\hline 6 & 7.2 & No zones. Polar bodies generally present. \\
\hline 8 & 9.6 & Most eggs lack zones. A few eggs have zones indicated. \\
\hline 10 & 12.0 & Three zones show plainly. \\
\hline 12 & 14.5 & Zones indicated in many eggs, never plain. \\
\hline 14 & 16.9 & Zones indicated in many eggs, never plain. \\
\hline 16 & 19.3 & $\begin{array}{l}\text { No zones generally (a few eggs have zones faintly } \\
\text { indicated). }\end{array}$ \\
\hline 18 & 21.7 & $\begin{array}{l}\text { No zones generally. The second polar body is form- } \\
\text { ing (or has formed). }\end{array}$ \\
\hline 20 & 24.1 & No zones. \\
\hline 22 & 26.5 & No zones. \\
\hline 24 & 28.9 & No zones. \\
\hline 26 & 31.3 & No zones. Pronuclei large, not apposed. \\
\hline 28 & 33.7 & $\begin{array}{l}\text { Zones show faintly in some eggs. Pronuclei large, } \\
\text { apposed. }\end{array}$ \\
\hline 30 & 36.1 & $\begin{array}{l}\text { Zones appear; usually they are not very sharply } \\
\text { marked off. Pronuclei large, apposed. }\end{array}$ \\
\hline 32 & 38,6 & $\begin{array}{l}\text { Zones show fairly plainly. Pronuclei usually broken } \\
\text { down. }\end{array}$ \\
\hline 34 & 41.0 & Zones show fairly plainly. \\
\hline 36 & 43.4 & $\begin{array}{l}\text { Zones usually show fairly plainly. In some eggs } \\
\text { they appear indistinct. }\end{array}$ \\
\hline 38 & 45.8 & $\begin{array}{l}\text { Some eggs show zones. Most eggs lack zones. A } \\
\text { few eggs are in the mitotic elongation stage. }\end{array}$ \\
\hline 40 & 48.2 & $\begin{array}{l}\text { No zones generally. Eggs have elongated and some } \\
\text { have begun to segment. }\end{array}$ \\
\hline $41^{\frac{1}{4}}$ & & $8 / 25$ segmented. \\
\hline $41^{\frac{3}{4}}$ & & $\begin{array}{l}15 / 25 \text { segmented. } 41 \frac{1}{2} \text { minutes taken as the time of } \\
\text { segmentation. }\end{array}$ \\
\hline $42 \frac{1}{2}$ & & $21 / 25$ segmented. \\
\hline
\end{tabular}


TABLE 3

Five-second tests. Eggs fertilized at 12.15 p.m. Temperature at 12.18 p.m., 22.0 $0^{\circ}$; at 1.10 p.m., $23.6^{\circ}$

\begin{tabular}{|c|c|c|}
\hline $\begin{array}{l}\text { ACTUAL TIME } \\
\text { AFTER } \\
\text { FERTILIZATION }\end{array}$ & $\underset{\text { TTMME }}{\text { STANDARD }}$ & OBSERVATIONS \\
\hline minutes & minutes & \\
\hline $2 \frac{1}{2}$ & 2.2 & Three zones show plainly. \\
\hline $5 \frac{1}{2}$ & 4.8 & Most eggs lack zones. Some eggs show them. \\
\hline 8 & 7.0 & Eggs generally lack zones. \\
\hline $11 \frac{1}{2}$ & 10.1 & No zones. \\
\hline 14 & 12.3 & No zones. \\
\hline $16 \frac{1}{2}$ & 14.5 & No zones. \\
\hline $18 \frac{1}{2}$ & 16.2 & No zones. \\
\hline 21 & 18.4 & Zones appear in many eggs, others lack them. \\
\hline $23 \frac{1}{2}$ & 20.6 & Zones appear generally. \\
\hline 26 & 22.8 & Zones in some eggs, others lack them. \\
\hline $28 \frac{1}{2}$ & 25.0 & No zones. \\
\hline $30 \frac{1}{2}$ & 26.8 & No zones. \\
\hline 33 & 29.0 & No zones. \\
\hline 36 & 31.6 & No zones. \\
\hline $39 \frac{1}{2}$ & 34.6 & No zones. Pronuclei large. \\
\hline $42 \frac{1}{2}$ & 37.3 & No zones show clearly. Some show zones indistinctly. \\
\hline 45 & 39.5 & Eggs generally show zones. \\
\hline $47 \frac{3}{4}$ & 41.9 & Eggs generally show zones. \\
\hline $50 \frac{1}{2}$ & 44.3 & No zones. \\
\hline 53 & 46.5 & $\begin{array}{l}\text { No zones. Eggs generally in mitotic elongation } \\
\text { stage. }\end{array}$ \\
\hline 56 & & $13 / 40$ segmented. \\
\hline 58 & & $\begin{array}{l}21 / 31 \text { segmented. } 57 \text { minutes taken as the time of } \\
\text { segmentation. }\end{array}$ \\
\hline 59 & & $83 / 100$ segmented. \\
\hline
\end{tabular}


TABLE 4

Six second tests. Eggs fertilized at 11.00 a.m. Temperature at 10.59 a.m., $28.4^{\circ}$; at 11.52 a.m., $26.4^{\circ}$

\begin{tabular}{|c|c|c|}
\hline $\begin{array}{l}\text { ACTUAL TIME } \\
\text { AFTER } \\
\text { EERTILIZATION }\end{array}$ & $\underset{\text { TIME }}{\text { ETANDARD }}$ & OBBERYATIONA \\
\hline minutes & minutes & \\
\hline $2 \frac{1}{2}$ & 2.6 & Three zones show plainly. \\
\hline 5 & 5.3 & $\begin{array}{l}\text { Zones when present are indistinet and not sharply } \\
\text { marked off. }\end{array}$ \\
\hline $7 \frac{1}{2}$ & 7.9 & No zones. Eggs have polar bodies. \\
\hline 10 & 10.5 & No zones. \\
\hline 12 & 12.6 & $\begin{array}{l}\text { Zones indicated. The dark zone is often plain but } \\
\text { the pigment zone does not show. }\end{array}$ \\
\hline 14 & 14.7 & $\begin{array}{l}\text { Zones show generally. The pigment zone is not } \\
\text { always distinct. }\end{array}$ \\
\hline 16 & 16.8 & Three zones show plainly. \\
\hline 18 & 18.9 & Three zones show plainly. \\
\hline 20 & 21.1 & $\begin{array}{l}\text { Dark zone sharply defined. Pigment zone usually not } \\
\text { sharply defined. }\end{array}$ \\
\hline 22 & 23.2 & No zones. \\
\hline 24 & 25.3 & No zones. \\
\hline 26 & 27.4 & No zones generally (a few exceptions). \\
\hline 28 & 29.5 & No zones generally (a few exceptions). \\
\hline 30 & 31.6 & No zones. \\
\hline $32 \frac{1}{2}$ & 34.2 & No zones. \\
\hline 35 & 36.8 & Zones appear. They are not very sharply defined. \\
\hline 37 & 38.9 & Three zones show plainly. \\
\hline 39 & 41.1 & Three zones show plainly. \\
\hline 41 & 43.2 & Three zones show plainly. \\
\hline 43 & 45.3 & $\begin{array}{l}\text { Many eggs show zones; in these the zones are not as } \\
\text { sharply defined as in last test. Some eggs lack zones. }\end{array}$ \\
\hline 45 & 47.4 & $\begin{array}{l}\text { Some eggs with zones. Most eggs in mitotic elonga- } \\
\text { tion stage or with cleavage furrow beginning. These } \\
\text { lack zones. }\end{array}$ \\
\hline $47 \frac{1}{4}$ & & $7 / 25$ segmented. \\
\hline $47 \frac{3}{4}$ & & $\begin{array}{l}13 / 25 \text { segmented. } 47 \frac{1}{2} \text { minutes taken as the time of } \\
\text { segmentation. }\end{array}$ \\
\hline 49 & & $19 / 25$ segmented. \\
\hline 50 & & $23 / 25$ segmented. \\
\hline
\end{tabular}


TABLE 5

Seven-second tests. Eggs fertilized at 5.20 p.m. Temperature at 5.19 p.m., $20.3^{\circ}$; at 6.17 p.m., $24 .^{\circ}$

\begin{tabular}{|c|c|c|}
\hline $\begin{array}{l}\text { ACTUAL TIME } \\
\text { AATER } \\
\text { WERTILIZATION }\end{array}$ & $\begin{array}{l}\text { STANDARD } \\
\text { TIME }\end{array}$ & ogservations \\
\hline minutes & minutes & \\
\hline 2 & 1.9 & Three zones show plainly. \\
\hline 4 & 3.7 & $\begin{array}{l}\text { Three zones show, but not as plainly as in previous } \\
\text { test. }\end{array}$ \\
\hline 6 & 5.6 & $\begin{array}{l}\text { Most eggs show three zones. In some eggs these zones } \\
\text { are indistinct. }\end{array}$ \\
\hline 8 & 7.4 & $\begin{array}{l}\text { Generally no zones. Polar body beginning to appear } \\
\text { in one egg. }\end{array}$ \\
\hline 10 & 9.3 & No zones, polar bodies general. \\
\hline 12 & 11.1 & No zones. \\
\hline 14 & 13.0 & No zones (perhaps they are indicated in a few eggs). \\
\hline 16 & 14.8 & Many eggs show zones; these are not very distinct. \\
\hline 18 & 16.7 & Three zones show plainly in most eggs. \\
\hline 20 & 18.5 & Three zones show plainly. \\
\hline 22 & 20.4 & Three zones show plainly. \\
\hline 24 & 22.2 & $\begin{array}{l}\text { Some eggs show zones, others lack zones or show them } \\
\text { indistinctly. }\end{array}$ \\
\hline 26 & 24.1 & $\begin{array}{l}\text { No zones generally. Many eggs are elongated, in- } \\
\text { dicating mitotic elongation for second maturation } \\
\text { division. }\end{array}$ \\
\hline 28 & 25.9 & No zones generally (a few exceptions). \\
\hline 30 & 27.8 & No zones generally (a few exceptions). \\
\hline 32 & 29.6 & No zones generally. \\
\hline 34 & 31.5 & $\begin{array}{l}\text { No zones generally. Pronuclei fairly large, not ap- } \\
\text { posed. }\end{array}$ \\
\hline 36 & 33.3 & $\begin{array}{l}\text { No zones generally. Pronuciei large, apposed or } \\
\text { nearly apposed. }\end{array}$ \\
\hline 38 & 35.2 & $\begin{array}{l}\text { Zones appear indistinctly in most eggs; in a few eggs } \\
\text { they are distinct. Pronuclei large, apposed. }\end{array}$ \\
\hline 40 & 37.0 & $\begin{array}{l}\text { The three zones show quite distinctly. Pronuclei are } \\
\text { still present. }\end{array}$ \\
\hline 42 & 38.9 & $\begin{array}{l}\text { Three zones show plainly. Pronuclei have broken } \\
\text { down in most eggs. }\end{array}$ \\
\hline 44 & 40.7 & Three zones show plainly. \\
\hline 46 & 42.6 & $\begin{array}{l}\text { Three zones show plainly, they appear sharper and } \\
\text { clearer than at any previous test. }\end{array}$ \\
\hline 48 & 44.4 & In most eggs, three zones distinct; in some, no zones. \\
\hline 50 & 46.3 & $\begin{array}{l}\text { A few eggs show three zones, the majority lack zones } \\
\text { or show them indistinctly. Mitotic elongation } \\
\text { is beginning in some eggs. }\end{array}$ \\
\hline $\begin{array}{l}52 \\
53 \frac{1}{2} \\
54 \\
54 \frac{1}{4} \\
55\end{array}$ & 48.1 & $\begin{array}{l}\text { No zones. Eggs generally show mitotic elongation. } \\
3 / 25 \text { segmented. } \\
10 / 25 \text { segmented. This taken as time of segmentation. } \\
19 / 25 \text { segmented. } \\
23 / 25 \text { segmented. }\end{array}$ \\
\hline
\end{tabular}


TABLE 6

Eight-second tests. Temperature, $24.2^{\circ}$

\begin{tabular}{|c|c|c|}
\hline $\begin{array}{c}\text { ACTDALTIME } \\
\text { AFTER } \\
\text { FERTILIZATION }\end{array}$ & $\begin{array}{l}\text { STANDARD } \\
\text { TIME }\end{array}$ & OBSERVATIONS \\
\hline minutes & minutes & \\
\hline 2 & 1.8 & Zones show. \\
\hline 5 & 4.6 & Dark zone shows, but pigment zone does not. \\
\hline $7 \frac{1}{2}$ & 6.9 & No zones. Polar bodies just beginning to be lifted off. \\
\hline 10 & 9.2 & No zones. \\
\hline 12 & 11.0 & No zones. \\
\hline 14 & 12.8 & Most eggs lack zones, a very few show dark zone. \\
\hline 16 & 14.7 & Three zones show plainly. \\
\hline 18 & 16.5 & Three zones show plainly. \\
\hline 20 & 18.3 & Dark zone plain, pigment zone not so distinct. \\
\hline $22 \frac{1}{2}$ & 20.6 & $\begin{array}{l}\text { Most eggs with zones not very distinet. A few eggs } \\
\text { lack zones completely. }\end{array}$ \\
\hline 26 & 23.9 & No zones. \\
\hline 28 & 25.7 & No zones. \\
\hline 30 & 27.5 & No zones. \\
\hline $32 \frac{1}{2}$ & 29.8 & No zones. \\
\hline 35 & 32.1 & No zones. Pronuclei large. \\
\hline $37 \frac{1}{2}$ & 34.4 & No zones. Pronuclei large and close to each other. \\
\hline 40 & 36.7 & $\begin{array}{l}\text { In many eggs zones show quite distinctly. These } \\
\text { apparently have pronuclei broken down. Some } \\
\text { eggs lack zones. }\end{array}$ \\
\hline $42 \frac{3}{4}$ & 39.2 & Three zones show plainly. \\
\hline $44_{4}^{\frac{3}{4}}$ & 41.1 & Three zones show plainly. \\
\hline $47 \frac{1}{2}$ & 43.6 & Three zones show plainly. \\
\hline $50 \frac{1}{2}$ & 46.3 & No zones. \\
\hline $53 \frac{1}{2}$ & & $3 / 50$ segmented. \\
\hline $53 \frac{3}{4}$ & & $7 / 50$ segmented. \\
\hline $54 \frac{1}{2}$ & & $9 / 50$ segmented. \\
\hline $54 \frac{3}{4}$ & & $\begin{array}{l}14 / 25 \text { segmented. } 54 \frac{1}{2} \text { minutes taken as the time of } \\
\text { segmentation. }\end{array}$ \\
\hline $55 \frac{1}{2}$ & & $21 / 25$ segmented. \\
\hline
\end{tabular}


TABLE 7

Ten-second tests. Eggs fertitized at 6.45 p. m. Temperature at 6.57 p.m., 27.4 $4^{\circ}$; at 7.28 p.m., $27.4^{\circ}$

\begin{tabular}{|c|c|c|}
\hline $\begin{array}{l}\text { ACTUAL TIME } \\
\text { ATER } \\
\text { FERILIZATION }\end{array}$ & $\underset{T M M E}{\operatorname{STANDARD}}$ & omservations \\
\hline minutes & minutes & \\
\hline 2 & 2.5 & Three zones show plainly. \\
\hline 4 & 4.9 & $\begin{array}{l}\text { In most eggs three zones show plainly, in some they } \\
\text { are not so plain. }\end{array}$ \\
\hline 7 & 8.6 & $\begin{array}{l}\text { Most eggs lack zones, these have first polar body. A } \\
\text { few with zones lack polar body. }\end{array}$ \\
\hline 10 & 12.3 & Three zones show plainly in most eggs. \\
\hline 13 & 16.0 & Three zones show plainly. \\
\hline 15 & 18.5 & $\begin{array}{l}\text { Three zones show plainly in some eggs. Other eggs } \\
\text { lack zones. }\end{array}$ \\
\hline $17 \frac{1}{2}$ & 21.6 & Zones are indicated, but are not sharply defined. \\
\hline $20 \frac{1}{2}$ & 25.3 & No zones. \\
\hline 23 & 28.4 & No zones. \\
\hline $25 \frac{1}{2}$ & 31.4 & No zones. \\
\hline 28 & 34.5 & $\begin{array}{l}\text { Most eggs lack zones, a few show them. Pronucle } \\
\text { large. }\end{array}$ \\
\hline $30 \frac{1}{2}$ & 37.7 & Three zones show plainly. \\
\hline $32 \frac{1}{2}$ & 40.1 & Three zones show plainly. \\
\hline $34 \frac{1}{2}$ & 42.6 & Three zones show plainly. \\
\hline 37 & 45.7 & $\begin{array}{l}\text { Three zones show plainly in many eggs. Some eggs } \\
\text { are in mitotic elongation stage and these lack zones }\end{array}$ \\
\hline $\begin{array}{l}39 \\
40 \frac{1}{2}\end{array}$ & 48.1 & $\begin{array}{l}\text { No zones. Segmentation beginning in some. } \\
10 / 25 \text { segmented. This taken as the time of segmenta- } \\
\text { tion. }\end{array}$ \\
\hline 41 & & $17 / 25$ segmented. \\
\hline $41 \frac{1}{2}$ & & $22 / 25$ segmented. \\
\hline $42 \frac{1}{2}$ & & $23 / 25$ segmented. \\
\hline
\end{tabular}


TABLE 8

Twelve-second tests. Eggs fertilized at 3.55 p.m. Temperature at 3.51 p.m., $25.6^{\circ}$

\begin{tabular}{|c|c|c|}
\hline $\begin{array}{l}\text { ACTUAL TIME } \\
\text { ARTER } \\
\text { FEHTIIIZATION }\end{array}$ & $\begin{array}{l}\text { STANDARD } \\
\text { TLMEI }\end{array}$ & OBEKEYYATIONB \\
\hline minutes & minutes & \\
\hline 2 & 2.2 & Three zones show plainly. \\
\hline 5 & 5.6 & $\begin{array}{l}\text { Some eggs with zones distinct, others with dark zone } \\
\text { fairly sharp, but with pigment zone not well marked } \\
\text { off. }\end{array}$ \\
\hline $8 \frac{1}{2}$ & 9.6 & No zones. Polar bodies generally present. \\
\hline $11 \frac{1}{2}$ & 12.9 & $\begin{array}{l}\text { Three zones generally present. Pigment zone not } \\
\text { always sharply defined. }\end{array}$ \\
\hline 14 & 15.7 & Three zones show plainly. \\
\hline $16 \frac{1}{2}$ & 18.5 & Three zones show plainly. \\
\hline 19 & 21.3 & $\begin{array}{l}\text { Three zones show plainly in some eggs. In others } \\
\text { only the dark zone is clearly defined. }\end{array}$ \\
\hline 24 & 27.0 & $\begin{array}{l}\text { No zones (a few exceptions show indications of a dark } \\
\text { zone). }\end{array}$ \\
\hline $26 \frac{1}{2}$ & 29.8 & No zones. \\
\hline 29 & 32.6 & $\begin{array}{l}\text { Most eggs without zones. Zones are indicated in a } \\
\text { few. Pronuclei large. }\end{array}$ \\
\hline $31 \frac{1}{2}$ & 35.4 & $\begin{array}{l}\text { Zones appear, but they are not very sharply delimited. } \\
\text { The pronuclei are large and apposed. }\end{array}$ \\
\hline 34 & 38.2 . & Three zones show plainly. \\
\hline $36 \frac{1}{2}$ & 41.0 & Three zones show plainly. \\
\hline $38 \frac{1}{2}$ & 43.3 & Three zones show plainly. \\
\hline 41 & 46.1 & Majority lack zones. Some have zones indicated. \\
\hline 43 & 48.3 & No zones. Cleavage furrows beginning. \\
\hline $44 \frac{1}{2}$ & & $\begin{array}{l}10 / 25 \text { segmented. This taken as the time of segmen- } \\
\text { tation. }\end{array}$ \\
\hline 45 & & $20 / 25$ segmented. \\
\hline $45 \frac{1}{2}$ & & $23 / 25$ segmented. \\
\hline $45 \frac{3}{4}$ & & $25 / 25$ segmented. \\
\hline
\end{tabular}


TABLE 9

Fourteen-second tests. Eggs fertilized at 10.50 a.m. Temperature at 10.49 a.m., $19.9^{\circ}$; at 12.06 p.m., $22.8^{\circ}$

\begin{tabular}{|c|c|c|}
\hline $\begin{array}{l}\text { ACTUAL TIME } \\
\text { AFTER } \\
\text { FERTIIIZATION }\end{array}$ & $\begin{array}{l}\text { STANDARD } \\
\text { TIME }\end{array}$ & OBSERvations \\
\hline minutes & minutes & \\
\hline 2 & 1.4 & Three zones show plainly. \\
\hline $4 \frac{1}{2}$ & 3.2 & $\begin{array}{l}\text { Three zones show plainly (in some they are not as } \\
\text { sharply defined as in previous test). }\end{array}$ \\
\hline 7 & 4.9 & $\begin{array}{l}\text { Three zones show generally, but the pigment zone is } \\
\text { not sharply marked off. }\end{array}$ \\
\hline 10 & 7.0 & $\begin{array}{l}\text { Zones are either not present or if present they are } \\
\text { indicated rather than sharply defined. }\end{array}$ \\
\hline 13 & 9.1 & No zones. Polar bodies generally present. \\
\hline $15 \frac{1}{2}$ & 10.9 & No zones. \\
\hline 18 & 12.7 & Zones indicated in some eggs. \\
\hline $20 \frac{1}{2}$ & 14.4 & $\begin{array}{l}\text { Three zones appear plainly in some eggs. In others } \\
\text { only the dark zone appears. }\end{array}$ \\
\hline 23 & 16.2 & Three zones show plainly. \\
\hline $25 \frac{1}{2}$ & 18.0 & Three zones show plainly. \\
\hline 28 & 19.7 & Three zones show plainly. \\
\hline $30_{2}^{1}$ & 21.5 & $\begin{array}{l}\text { Three zones show plainly in most eggs, in some they } \\
\text { are not sharply defined. }\end{array}$ \\
\hline 33 & 23.3 & $\begin{array}{l}\text { Most eggs lack zones, a few show them. Eggs have } \\
\text { one, but not two polar bodies. }\end{array}$ \\
\hline 35 & 24.6 & $\begin{array}{l}\text { No zones. A few eggs were examined carefully and } \\
\text { showed second polar body forming. }\end{array}$ \\
\hline 38 & 26.8 & $\begin{array}{l}\text { Zones appear in many eggs. They are not sharply } \\
\text { defined. }\end{array}$ \\
\hline $40 \frac{1}{2}$ & 28.5 & $\begin{array}{l}\text { Zones do not show plainly. Sometimes the dark zone } \\
\text { appears, but the pigment zone does not. }\end{array}$ \\
\hline 44 & 31.0 & No zones. \\
\hline $46 \frac{1}{2}$ & 32.8 & No zones. \\
\hline 49 & 34.5 & Zones indicated, never distinct. \\
\hline $51 \frac{1}{2}$ & 36.3 & $\begin{array}{l}\text { Zones appear, they are not very distinet. The pro- } \\
\text { nuclei are very large. }\end{array}$ \\
\hline 54 & 38.0 & Three zones show plainly. \\
\hline $56 \frac{1}{2}$ & 39.8 & Three zones show plainly. \\
\hline 59 & 41.5 & Three zones show plainly. \\
\hline $61 \frac{1}{2}$ & 43.3 & $\begin{array}{l}\text { Three zones generally show plainly (a few exceptions } \\
\text { in which zones were not very sharply marked off). }\end{array}$ \\
\hline $63 \frac{1}{2}$ & 44.7 & $\begin{array}{l}\text { Three zones generally show plainly (a few exceptions } \\
\text { in which zones were not very sharply marked off). }\end{array}$ \\
\hline 66 & 46.5 & $\begin{array}{l}\text { A few eggs show zones plainly. Most eggs are in mito- } \\
\text { tic elongation stage and lack zones. }\end{array}$ \\
\hline $68 \frac{1}{2}$ & 48.2 & No zones. Eggs generally in mitotic elongation stage. \\
\hline $\begin{array}{l}70 \\
70 \frac{1}{3}\end{array}$ & & $3 / 25$ segmented. \\
\hline $70 \frac{1}{2}$ & & 7/25 segmented. \\
\hline 71 & & $\begin{array}{l}15 / 25 \text { segmented. This taken as the time of segmen- } \\
\text { tation. }\end{array}$ \\
\hline $71 \frac{1}{2}$ & & 19/25 segmented. \\
\hline $72 \frac{1}{2}$ & & $22 / 25$ segmented. \\
\hline $73 \frac{1}{2}$ & & $23 / 25$ segmented. \\
\hline
\end{tabular}


TABLE 10

Sixteen-second tests. Eggs fertilized at 11.20 a.m. Temperature at 11.18 a.m., $19.0^{\circ}$; at 12.34 p.m., $23.4^{\circ}$

\begin{tabular}{|c|c|c|}
\hline $\begin{array}{l}\text { ACTUAI TIME } \\
\text { ATTER } \\
\text { FERTILIZATION }\end{array}$ & $\begin{array}{l}\text { STANDARD } \\
\text { TIME }\end{array}$ & OBSERVATIONS \\
\hline minutes & minutes & \\
\hline 2 & 1.5 & Three zones show plainly. \\
\hline $7 \frac{1}{2}$ & 5.5 & $\begin{array}{l}\text { Three zones show plainly in most eggs. A few ex- } \\
\text { ceptions. }\end{array}$ \\
\hline 10 & 7.4 & Zones show, but are generally indistinet. \\
\hline $12 \frac{1}{2}$ & 9.2 & $\begin{array}{l}\text { Zones not distinct. Dark zone indicated, no pigment } \\
\text { zone. }\end{array}$ \\
\hline $15 \frac{1}{2}$ & 11.4 & Zones show indistinetly. \\
\hline 18 & 13.2 & Three zones show plainly. \\
\hline 20 & 14.7 & Three zones show plainly. \\
\hline $22 \frac{1}{2}$ & 16.5 & Three zones show plainly. \\
\hline 25 & 18.4 & Three zones show plainly. \\
\hline $27 \frac{1}{2}$ & 20.2 & Three zones show plainly. \\
\hline 30 & 22.1 & $\begin{array}{l}\text { Zones appear, but are not as sharply marked off as in } \\
\text { previous test. }\end{array}$ \\
\hline 33 & 24.3 & $\begin{array}{l}\text { Elongated eggs (i.e., those giving off second polar } \\
\text { body) lack zones or have them barely indicated. } \\
\text { Spherical eggs show zones. }\end{array}$ \\
\hline $36 \frac{1}{2}$ & 26.8 & $\begin{array}{l}\text { Zones are indicated, but do not show plainly. The } \\
\text { dark zone is more distinct than the pigment zone. }\end{array}$ \\
\hline 39 & 28.7 & $\begin{array}{l}\text { Zones not distinct, but often they can be made out. } \\
\text { The dark zone is. more plainly indicated than the } \\
\text { pigment zone. }\end{array}$ \\
\hline $41 \frac{1}{2}$ & 30.5 & Zones indicated, especially the dark zone. \\
\hline $44 \frac{1}{2}$ & 32.7 & No zones generally, a few exceptions. \\
\hline $47 \frac{1}{2}$ & 34.9 & Zones indicated generally. Pronuclei large. \\
\hline 50 & 36.8 & $\begin{array}{l}\text { Three zones appear distinctly, although they are often } \\
\text { not very sharply marked off. Pronuclei large. }\end{array}$ \\
\hline $52 \frac{1}{2}$ & 38.6 & $\begin{array}{l}\text { Three zones show plainly. Pronuclei large, their } \\
\text { boundaries fading. }\end{array}$ \\
\hline 55 & 40.5 & $\begin{array}{l}\text { Three zones show plainly. Pronuclei not large or } \\
\text { prominent; they have probably broken down. }\end{array}$ \\
\hline $57 \frac{1}{2}$ & 42.3 & Three zones show plainly. \\
\hline 60 & 44.1 & Three zones show plainly. \\
\hline $62 \frac{1}{2}$ & 46.0 & $\begin{array}{l}\text { Some eggs show zones. Many eggs in mitotic elon- } \\
\text { gation stage and lack zones. }\end{array}$ \\
\hline 65 & 47.8 & $\begin{array}{l}\text { Most eggs in mitotic elongation stage or beginning } \\
\text { segmentation. They lack zones. }\end{array}$ \\
\hline 67 & & $5 / 25$ segmented. \\
\hline $67 \frac{1}{2}$ & & $8 / 25$ segmented. \\
\hline $67 \frac{3}{4}$ & & $\begin{array}{l}13 / 25 \text { segmented. } 68 \mathrm{~min} \text {. taken as time of segmenta- } \\
\text { tion. }\end{array}$ \\
\hline $68 \frac{1}{2}$ & & $18 / 25$ segmented. \\
\hline 69 & & $20 / 25$ \\
\hline 70 & & $22 / 25$ \\
\hline 71 & & $23 / 25$ \\
\hline
\end{tabular}


TABLE II

Eighteen-second tests. Eggs fertilized at $12 \mathrm{~m}$. Temperature at 12.07 p.m., $23.0^{\circ}$; at 1.00 p.m., $24.5^{\circ}$

\begin{tabular}{|c|c|c|}
\hline $\begin{array}{c}\text { ACTUAL TMME } \\
\text { AFTER } \\
\text { FERTILIZATION }\end{array}$ & $\underset{\substack{\text { STANDARD } \\
\text { TIME }}}{\text { STE }}$ & OBSERVATIONS \\
\hline minutes & minutes & \\
\hline 2 & 1.7 & Zones show very plainly. \\
\hline 5 & 4.2 & Three zones show very plainly. \\
\hline 8 & 6.7 & Three zones show very plainly. \\
\hline 11 & 9.2 & $\begin{array}{l}\text { Three zones show plainly, but eggs with polar body } \\
\text { have hyaline zone clouded over with granules. }\end{array}$ \\
\hline 14 & 11.8 & $\begin{array}{l}\text { Three zones show plainly, hyaline zone clouded with } \\
\text { granules. }\end{array}$ \\
\hline 17 & 14.3 & Three zones show plainly. \\
\hline $19 \frac{1}{2}$ & 16.4 & Three zones show plainly. \\
\hline 22 & 18.5 & Three zones show plainly. \\
\hline $24 \frac{1}{2}$ & 20.6 & $\begin{array}{l}\text { Three zones show plainly, In some eggs hyaline zone } \\
\text { is clouded with granules. }\end{array}$ \\
\hline 27 & 22.7 & $\begin{array}{l}\text { Three zones show plainly. In some (elongated eggs), } \\
\text { hyaline zone is somewhat cloudy. }\end{array}$ \\
\hline 30 & 25.2 & $\begin{array}{l}\text { Three zones show plainly. A few with hyaline zone } \\
\text { cloudy. }\end{array}$ \\
\hline 33 & 27.7 & Three zones show. Hyaline zone usually cloudy. \\
\hline 36 & 30.3 & $\begin{array}{l}\text { Three zones show. Hyaline zone clouded with gran- } \\
\text { ules. Pronuclei fairly large. }\end{array}$ \\
\hline 39 & 32.8 & $\begin{array}{l}\text { Three zones show. Hyaline zone somewhat clouded. } \\
\text { Pronuclei large, apposed. }\end{array}$ \\
\hline 42 & 35.3 & $\begin{array}{l}\text { Three zones show plainly, hyaline zone not clouded. } \\
\text { Pronuclei large, apposed. }\end{array}$ \\
\hline 45 & 37.8 & $\begin{array}{l}\text { Three zones show plainly. In some eggs pronuclei } \\
\text { are present, in others they have disappeared. }\end{array}$ \\
\hline 48 & 40.3 & $\begin{array}{l}\text { Three zones show plainly. In most eggs pronuclei } \\
\text { have disappeared. }\end{array}$ \\
\hline $50 \frac{1}{2}$ & 42.4 & Three zones show plainly. \\
\hline 53 & 44.5 & $\begin{array}{l}\text { Three zones show plainly in most eggs. A few are in } \\
\text { mitotic elongation stage and have zones indicated. }\end{array}$ \\
\hline $55 \frac{1}{2}$ & 46.6 & $\begin{array}{l}\text { Elongated eggs and dividing eggs have zones indicated. } \\
\text { They are not distinct, but can be made out. }\end{array}$ \\
\hline $\begin{array}{l}59 \\
59 \frac{1}{2}\end{array}$ & & $\begin{array}{l}7 / 25 \text { segmented. } \\
10 / 25 \text { segmented. This taken as the time of segmen- } \\
\text { tation. }\end{array}$ \\
\hline 60 & & $18 / 25$ segmented. \\
\hline 62 & & $\begin{array}{l}17 / 25 \text { segmented. Thus only about } 70 \text { per cent seg- } \\
\text { mented. }\end{array}$ \\
\hline
\end{tabular}


TABLE 12

Eighteen-second tests. Eggs fertilized at 11.40 a.m. Temperature at 11.42 a.m., $24.3^{\circ}$; at 12.30 a.m., $24.0^{\circ}$

\begin{tabular}{|c|c|c|}
\hline $\begin{array}{c}\text { ACTUAL TMME } \\
\text { AFTER } \\
\text { FERTILIZATION }\end{array}$ & $\begin{array}{l}\text { STANDARD } \\
\text { TIME }\end{array}$ & OBSERVATtONS \\
\hline minutes & minutes & \\
\hline 3 & 3.1 & Three zones show plainly. \\
\hline 6 & 6.2 & $\begin{array}{l}\text { Many eggs show three zones plainly. In some eggs } \\
\text { hyaline zone is clouded with granules and sometimes } \\
\text { the pigment zone is not sharply marked off. }\end{array}$ \\
\hline 9 & 9.3 & $\begin{array}{l}\text { Dark zone and pigment zone show plainly, hyaline } \\
\text { zone clouded somewhat. }\end{array}$ \\
\hline 14 & 14.4 & Three zones show plainly. \\
\hline 22 & 22.7 & Zones appear, but are not always sharply marked off. \\
\hline 25 & 25.8 & $\begin{array}{l}\text { Dark zone sharply defined, pigment zone not sharply } \\
\text { marked off. }\end{array}$ \\
\hline 29 & 29.9 & $\begin{array}{l}\text { Zones do not show distinctly. They are indicated } \\
\text { rather than sharply marked off. Dark zone plainer } \\
\text { than pigment zone. }\end{array}$ \\
\hline $32 \frac{1}{2}$ & 33.5 & $\begin{array}{l}\text { Zones show more plainly than in previous test. Pro- } \\
\text { nuclei apposed, apparently fused. }\end{array}$ \\
\hline 36 & 37.1 & Three zones show plainly. \\
\hline 39 & 40.2 & Three zones show plainly. \\
\hline 41 & 42.3 & Three zones show plainly. \\
\hline 43 & 44.3 & $\begin{array}{l}\text { In most eggs, three zones show plainly. A few eggs } \\
\text { are elongated and in these there is no distinct pig- } \\
\text { ment zone; the dark zone appears, but is not well } \\
\text { marked off. }\end{array}$ \\
\hline $45^{\frac{\pi}{6}}$ & 47.2 & $\begin{array}{l}\text { Most of the eggs are elongated. These show no zones. } \\
\text { Other eggs show zones. }\end{array}$ \\
\hline $48 \frac{1}{2}$ & & $\begin{array}{l}24 / 50 \text { segmented. This taken as the time of segmen- } \\
\text { tation. }\end{array}$ \\
\hline $49 \frac{1}{2}$ & & $43 / 50$ segmented. \\
\hline
\end{tabular}

The tables show that the viscosity of the protoplasm undergoes marked changes. With the same centrifugal speed sometimes two seconds are sufficient to produce zones, sometimes sixteen seconds must elapse before zones appear. In the latter case obviously the velocity of the granules is only one-eighth as great. Since the viscosity varies inversely as the velocity (see previous discussion) this indicates an eight-fold increase in viscosity. The number of seconds necessary to cause the appearance of zones is thus an inverse measure of the velocity of granular 
movement and a direct measure of the viscosity. As shown previously it requires on the average two turns of the centrifuge handle (in two seconds) to produce zones in unfertilized eggs. An arbitrary scale of viscosity may be established and the viscosity of the protoplasm of unfertilized eggs can be taken as 2 . At a stage when sixteen turns are required to show zones the viscosity in terms of the arbitrary unit is 16 .

From the data in the tables a viscosity curve may be plotted. This is not, however, a simple matter. In general it is not possible to obtain direct readings of viscosity. Usually each test indicates that the viscosity of the protoplasm is above or below a certain value, but it does not show how much above or below. Moreover, there is often some variation in the eggs of a given lot, especially at times of transition. By noting for each test whether the viscosity is above or below a certain value a curve may be plotted by drawing a line above the one set of points and below the other set.

The following symbols were used in marking out points:

$V=$ Zones show plainly in all eggs. Viscosity above value at apex of $V$.

$\wedge=$ No zones appear in any eggs. Viscosity below value at apex of inverted $V$.

$\circ=$ Zones appear but indistinctly (usually dark zone plainer than pigment zone). Viscosity about equal to value at center of circle.

The above three symbols were combined in a variety of ways.

$x=$ Eggs with zones and without zones about equal in number.

$x=$ Most eggs with zones, a few without.

$x=$ Most eggs without zones, a few with.

In those cases where zones appeared indistinctly, the arc of a circle was added to the upper or lower portion of any of the last three symbols.

In the curve the abscissa represents time in minutes after fertilization. Of course for purposes of comparison the socalled 'standard time' had to be employed. The ordinates represent comparative viscosity values on the basis of the arbitrary scale mentioned above. 
Not all of the available data were included in the curve. All of the tests in table 12 were omitted. Introduction of these figures would not have changed the curve. One test is included which is not given in the tables. This is a twenty-second test made when 5 per cent of the eggs had segmented, hence about a minute before 50 per cent had segmented.

Curve and tables show that there are three times when the viscosity is high. These are just before the first polar body is given off, just before the second polar body is given off, and just before the completion of the first cleavage division. Thus in every case immediately before the division of the cell, there is an increase in viscosity. There is also a high viscosity in the early stages of each mitosis. Generally the completion of one division is bound up with the preparation for the next. As the first polar body is given off, the second maturation spindle begins to form, ${ }^{3}$ and the viscosity increase at this time is probably causally related to the appearance of the spindle. As the second polar body is given off there is again a viscosity increase. After the completion of the second maturation division the viscosity decreases slightly. It soon rises again in preparation for the cleavage spindle. As the cleavage spindle forms the viscosity drops and remains low until immediately before cleavage, when there is a sharp increase. In general the viscosity increase at the conclusion of each mitosis is directly related to the appearance of the spindle in the mitosis which follows. The concluding phases of one division are bound up with the initial phases of the next. The same sort of phenomenon was previously found to occur in successive cleavages in the sea-urchin egg.

\section{COMPARISONS WITH OTHER EGGS}

One point emphasized by the curve is the sudden increase in viscosity just before division. This increase preceded division by a very short interval. The curve shows the interval to be about two minutes or a trifle more. It must be remembered,

${ }^{3}$ The second maturation spindle is a new structure and does not result from a transformation of the first maturation spindle. This is shown by the observations of Griffin ('99), Lillie ('01), and various other observers. 


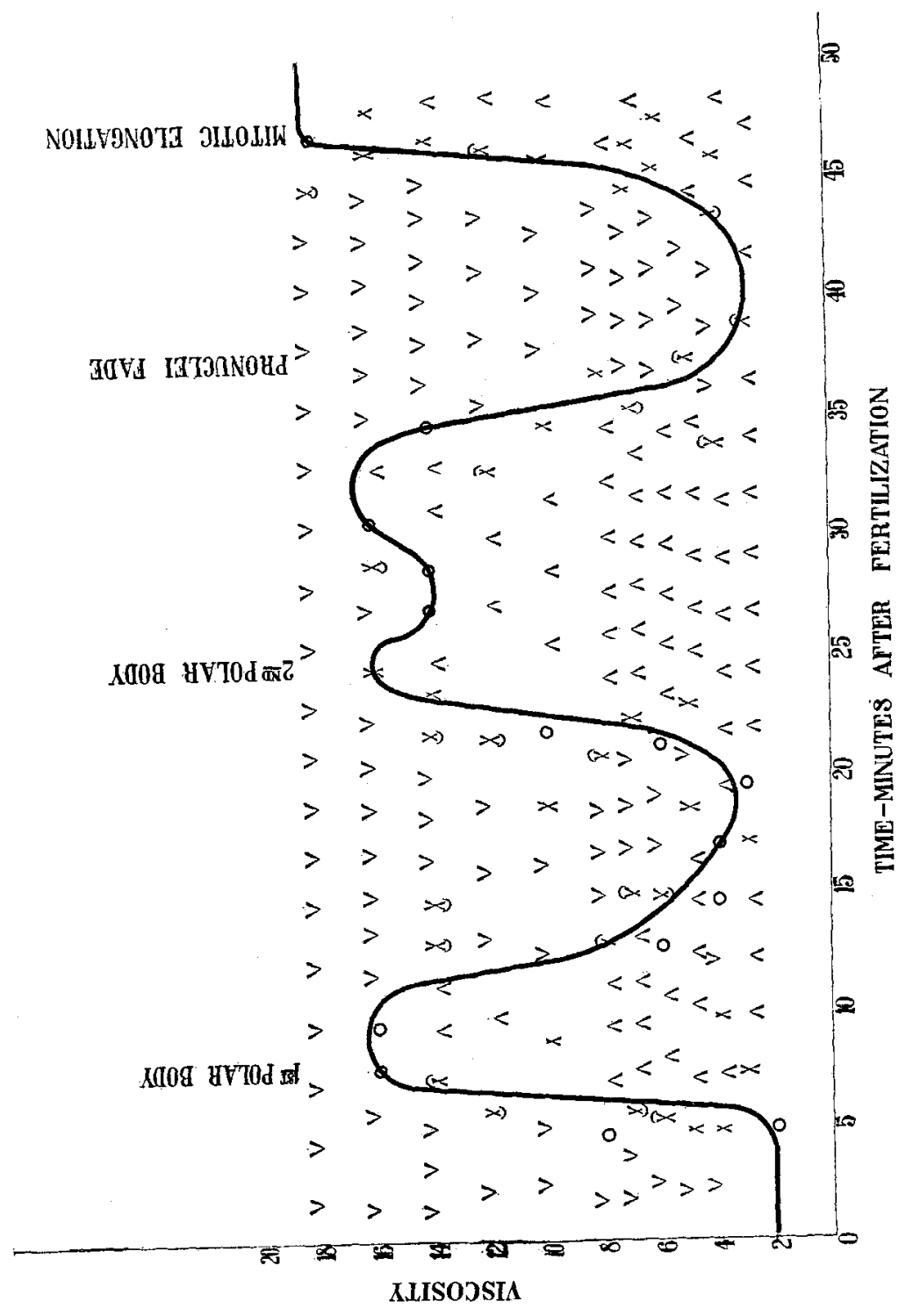

THE JOURNAL OF EXPERIMENTAL ZOÖLOGY, YOL. 34, No. 3 
however, that the time of cleavage is taken as the time of completed segmentation. The interval between viscosity increase and the first constriction of the cell is somewhat less. Obviously it is not much more than a minute.

In the sea-urchin egg a similar viscosity increase occurs just prior to division. This was indicated by some of my earlier data (cf., for example, the second table on page 216 of my 1920 paper). In earlier experiments, however, I was primarily interested in the viscosity increase which precedes the appearance of the spindle, and I did not study carefully the viscosity at the conclusion of mitosis. The intervals between tests were too large and, moreover, I took as the time of segmentation the time when the first eggs began to divide. It is much more exact to consider as the time of segmentation the moment when 50 per cent of the eggs have divided.

In Arbacia there is a rise in viscosity before division. This is shown conclusively by some tests made in 1919. In these tests an old-model Bausch \& Lomb centrifuge was used. It differs from the new model in having a shorter distance between the ends of the tubes, the radius of turn being $6 \mathrm{~cm}$.

June 30, 1919. Temperature $18.0^{\circ}$ at 10.58 A.M., $19.0^{\circ}$ at 11.42 A.M. Arbacia eggs were fertilized at five-minute intervals. In finger-bowl $A$ eggs were fertilized at 10.55 A.M., in $B$ at 11.00 A.M., and in $C$ at 11.05 A.M. At $12.10 \frac{1}{2}$ P.M. less than 1 per cent of the eggs in $A$ had begun segmentation. At $12.10 \frac{3}{4}$ P.M. eggs from $A$ and $B$ were centrifuged simultaneously in separate tubes, the high-speed handle being turned 30 times in 25 seconds. On examination of centrifuged eggs, the great majority of eggs from $A$ showed no hyaline zone, only about 10 to 20 per cent showing such a zone. The centrifuged eggs from $B$ all showed a hyaline zone plainly extending one-third of the distance through the egg.

At $12.17 \frac{1}{4}$ P.M. about 5 per cent of the eggs in $B$ were beginning to segment. Starting at $12.17 \frac{1}{2}$ P.M., eggs from $B$ and $C$ were centrifuged simultaneously, the high-speed handle being turned 30 times in 28 seconds. When eggs from $B$ were examined, there was no hyaline zone in any of the eggs. Eggs from $C$ all showed a hyaline zone, sometimes indistinct, sometimes extending a third of the distance through the egg.

Other tests gave similar results. Evidently just before division in Arbacia as in Cumingia there is a sudden increase in 
viscosity. This increase is of considerable importance and can of course be studied experimentally in the same manner that the viscosity increase in early prophase has been studied. This I propose to do in the near future.

The viscosity changes in Arbacia and Cumingia are absolutely parallel. In each case the appearance of a spindle is preceded by an increase in viscosity and followed by a decrease in viscosity. And in both eggs division of the cell is immediately preceded by a viscosity increase.

Besides the two eggs already mentioned the egg of Nereis was also studied. The Heteronereis form of Nereis limbata is common at Woods Hole. Its early development is well known through the studies of E. B. Wilson and of F. R. Lillie. The egg ' is immature at fertilization and three complete mitoses occur between fertilization and cleavage. In Nereis viscosity changes are not as sharply marked as in Cumingia or Arbacia and the egg is therefore more difficult to study. The Nereis egg is much larger than the eggs of the other two forms studied and it contains a considerable amount of yolk material. In proportion to the size of the egg, the spindle is small. We might expect that the viscosity changes associated with the appearance of the spindle would be masked in an egg when the spindle constitutes but a small fraction of the material of the egg.

A number of tests were made on the Nereis egg, but only one series is included here, these are given in table 13. All tests were made at a uniform centrifugal speed. The new-model Bausch \& Lomb centrifuge was used and the handle was turned five times in five seconds.

The table shows that in the immature Nereis egg the protoplasm is very stiff. Soon after fertilization and the disappearance of the germinal vesicle the viscosity decreases. Following this there are four times of viscosity increase, at $20,35,47$, and 65 minutes after fertilization. These viscosity increases presumably correspond with, 1) early prophase of first maturation division; 2) conclusion of first and early prophase of second maturation division; 3) conclusion of second maturation division and early prophase or cleavage mitosis; 4) conclusion of 
TA_3LE 13

Five-second tests. Nereis. Eggs fertilized at 10.55 a.m. Temperature at 11.11 a. m., $23.7^{\circ}$; at 12.20 p.m. $24.9^{\circ}$

\begin{tabular}{|c|c|}
\hline $\begin{array}{l}\text { TIME AFTER } \\
\text { FERTILIZATIOA }\end{array}$ & OBgERVATIONG \\
\hline 2 & No hyaline zone. Germinal vesicle present. \\
\hline 5 & No hyaline zone. Germinal vesicle present. \\
\hline 8 & No hyaline zone. Germinal vesicle nearly faded out. \\
\hline 11 & Hyaline zone beginning to appear. No germinal vesicle. \\
\hline 14 & $\begin{array}{l}\text { Hyaline zone appears plainly; it extends through about one-fifth of } \\
\text { egg. }\end{array}$ \\
\hline 17 & $\begin{array}{l}\text { Hyaline zone not pronounced. In some eggs it is absent; in others, } \\
\text { if present, it is indicated rather than shown plainly. }\end{array}$ \\
\hline 20 & Hyaline zone is absent generally (perhaps it is indicated in a few). \\
\hline 23 & Hyaline zone plain. About one-fifth. \\
\hline 26 & Hyaline zone appears plainly. \\
\hline 29 & Hyaline zone can be seen, but in most cases it is indistinet. \\
\hline 32 & Hyaline zone can be seen, it is generally narrow and not distinet. \\
\hline 35 & Generally no hyaline zone. \\
\hline 38 & $\begin{array}{l}\text { Hyaline zone present. It is generally narrow, but distinct and } \\
\text { clear. }\end{array}$ \\
\hline 41 & Hyaline zone plain, extending through one-fifth of egg. \\
\hline 44 & Hyaline zone narrow if present at all. \\
\hline 47 & No hyaline zone. \\
\hline 50 & No hyaline zone. \\
\hline 53 & No hyaline zone. \\
\hline 56 & $\begin{array}{l}\text { In many eggs hyaline zone is wide, clear, plain. It extends through } \\
\text { one-fourth of egg, and its inner margin is almost at the equator. }\end{array}$ \\
\hline 62 & Hyaline zone wide and clear as in previous test. \\
\hline 65 & No hyaline zone in any eggs. \\
\hline 67 & Segmentation beginning generally. \\
\hline $67 \frac{1}{2}$ & $5 / 50$ segmented completely. \\
\hline 68 & $8 / 25$ segmented completely. \\
\hline $68 \frac{1}{2}$ & $17 / 25$ segmented completely. \\
\hline 69 & $21 / 25$ segmented completely. \\
\hline 70 & $49 / 50$ segmented completely. \\
\hline
\end{tabular}

cleavage division. ${ }^{4}$ Of the first three periods of increased viscosity the third, as might be expected, is the longest. It corresponds to the second period of increased viscosity in the Cumingia egg.

${ }^{4}$ According to Wilson ('92), the first polar body is formed thirty-five to forty minutes after fertilization, the second polar body ten to twelve minutes later, and the egg divides after about seventy-five minutes. Evidently Wilson worked at about the temperature of the above experiment. At lower temperatures the times are of course longer. 


\section{DISCUSSION}

In the last few years the importance of protoplasmic viscosity changes has been increasingly realized. Of all the properties of protoplasm the fact that it is a viscous fluid is perhaps its most general characteristic. That a viscous colloidal fluid like protoplasm would probably undergo marked viscosity changes was obvious to biologists long ago. They tried to associate many activities of protoplasm with changes in its consistency. In spite of the numerous theories that were proposed, actual attempts at measuring viscosity have until recently been almost totally lacking. Without doubt, if marked changes in protoplasmic viscosity occur, they must play an important part in the mechanics of many vital processes. There is now actual evidence to show that great viscosity changes do occur in protoplasm.

In addition to the results obtained on marine ova, Seifriz ('18, '20) and Leblond ('19) have shown viscosity changes in plant protoplasm and Bayliss ('20) has shown similar changes in Amoeba. Various methods have been used. The gravity method was employed by Heilbronn ('14) and later by F. and G. Weber ('17). Leblond and Bayliss used the presence of Brownian movement as a criterion of viscosity.

All of the methods are not equally reliable. To my mind the microdissection method particularly is open to question. Kite ('13) was the originator of the method. According to Kite, all egg protoplasm was a highly viscous gel which scarcely varied its consistency. After my demonstration of the fluidity of unfertilized sea-urchin egg protoplasm and the change to a gel after fertilization ('15), Chambers was able to confirm these facts with microdissection. At first I was inclined to consider Chambers' work as a valuable support to my views of protoplasmic viscosity change. In my 1920 paper this point of view is brought out. But the recent publications of the microdissectionists have made me doubt the value of the method. Compare, for example, recent statements of Chambers and Seifriz. Chambers ('19) says: "The time of appearance of the amphiaster until completion of cleavage lasts from 10 to 
15 minutes. The increased $d^{5}$ viscosity of the egy during this amphiaster stage could be more easily demonstrated by the needle in the eggs of Echinarachnius and Cerebratulus than in those of Arbacia.

"After completion of the cleavage process, there are indications that the firmness of the cytoplasm persists in the two blastomeres while they are still more or less spherical."

These remarks of Chambers apparently apply to the seaurchin egg. Working on the same egg apparently without any knowledge of Chambers' 1919 paper, to which he does not refer, Seifriz ('20) finds: "With the first appearance of the amphiasters there is a pronounced decrease $e^{5}$ in viscosity of the central region of the cell, and this condition is maintained throughout the intermediate stages of divisions (from middle prophase to late anaphase)." Later he says, "with the completion of division we have in each daughter cell of the embryo a general protoplasmic consistency identical with that of the egg before fertilization."

Thus Chambers considers the amphiaster stage to involve increased viscosity and Seifriz regards it as involving a pronounced decrease in viscosity (at least of the central region). And whereas, after the first cleavage, Chambers finds high viscosity, Seifriz finds a viscosity identical with that of the unfertilized egg. It might be thought that the difference is due to a difference in the capability of the two investigators, but this does not appear to be the case. For whereas Seifriz is wholly wrong in stating that the viscosity just after cleavage is no greater than in the unfertilized egg, Chambers is equally wrong in stating that the amphiaster stage is a stage of high viscosity. Evidently the difficulty lies primarily with the method. No doubt it is too subjective. Apparently all evidence as to protoplasmic viscosity gained from microdissection should for the present at least be regarded as merely suggestive.

In this paper it has been shown that during mitosis viscosity changes occur in Cumingia and Nereis which are similar to those

${ }^{5}$ Italics mine. 
occurring in the sea-urchin egg. The latter perhaps presents a somewhat simpler case, for the first cleavage division does not immediately follow the maturation divisions. The viscosity changes that occur in the sea-urchin egg are probably typical of mitosis in general. There is a marked viscosity increase in early prophase, then a decrease, and finally an increase just before the cell divides. This final viscosity increase in the seaurchin egg continues as the early prophase viscosity increase of the second cleavage division. In Cumingia and Nereis the maturation divisions are related to each other and to the first cleavage division as the first cleavage division is related to the second in the sea-urchin egg. Apparently whenever two mitoses follow each other in rapid succession, the viscosity increase at the conclusion of the first provides the essential viscosity increase for the beginning of the second.

In the various eggs studied the viscosity changes are apparently not of the same magnitude. In Nereis they are not as great as in the other eggs. This is readily understood in the light of the fact that the Nereis egg is considerably larger and its mitotic spindle forms a much smaller fraction of the material of the egg.

The magnitude of the changes has been determined only for Cumingia. In this egg the viscosity rises from an arbitrary. value of 2 or 3 to a value of 16 , and then drops to about its original value. These changes are very rapid and the increase in viscosity just mentioned occurs within a minute or two. Such a sharp and sudden viscosity increase is almost conclusive evidence that a gelation or coagulation has occurred within the egg. This does not mean that the entire protoplasm has gelled. Presumably only a part is affected. It seems likely that were the entire protoplasmic mass of the egg to gel, the viscosity increase would be greater. It is more logical to assume, however, that only that portion of the cytoplasm which is concerned with spindle formation undergoes gelation. This is borne out by the fact that in the Nereis egg, in which the spindle forms a smaller fraction of the egg material, the viscosity change is not so great.

The mechanism of viscosity increase and decrease or gelation and solation within the cell is not yet understood. It might be 
thought that gelation was due to an abstraction of water and solation to an addition of water. In an earlier paper some evidence for the first view was presented. If solation were due to an addition of water, it would naturally be expected that the breakdown of the nucleus during mitosis would be the primary cause of solation. This is, however, not the case in Cumingia. In the curve shown in figure 1, after the second polar body has formed the viscosity remains high for the formation of the cleavage spindle. The viscosity then drops, but this drop occurs not after the breakdown of the pronuclei, but before the pronuclei have shown any visible indication of breakdown. Thus the return of the protoplasm to a more fluid state is apparently not due to a passage of water from nucleus to cytoplasm. As far as can be judged from the published figures of Morgan ('10), Jordan ('10), the time of liquefaction is approximately simultaneous with the appearance of the fully formed cleavage spindle. The above authors show the spindle appearing at a time when the pronuclei have grown to a large size. It is at this time that the drop in viscosity occurs. Further evidence that the liquefaction of the protoplasm is not due to a breakdown of the nucleus is indicated in Cumingia by the fact that in this egg the protoplasm becomes more fluid immediately after the second maturation spindle is formed. In this case no nuclear breakdown occurs.

It appears that whenever a spindle forms there is a return to a fluid condition. It is as though the spindle were coagulated out of the cytoplasm. Thus the curve of viscosity at the time of spindle formation is like the viscosity curve of a coagulating albumen solution. As coagulation occurs in such a solution the viscosity suddenly increases greatly, after coagulation has been completed there is a sharp drop in viscosity. The first part of the Cumingia viscosity curve resembles the curve of a coagulating albumen solution given in Dunstan and Thole's 'Viscosity of liquids' (fig. 9). Of course, this resemblance may be superficial. At present, however, the idea that the spindle coagulates out probably offers the best interpretation of the viscosity changes which precede and follow spindle formation. 


\section{SUMMARY}

1. The centrifuge method of measuring protoplasmic viscosity is a perfectly sound method from a physical standpoint. With it quantitative data may be obtained.

2. The velocity of granular movement under the influence of centrifugal force was taken as a measure of the viscosity.

3. The viscosity of Cumingia egg protoplasm was plotted for the period between fertilization and first cleavage. The data may be presented in the form of a curve (fig. 1).

4. In both maturation divisions and cleavage, appearance of the spindle is always preceded by a sharp viscosity increase and followed by a sharp viscosity decrease.

5. At the conclusion of mitosis, within a minute or two before the division of the cell is completed, a sharp increase in viscosity occurs.

6 . When two mitoses follow each other, the concluding viscosity increase of the first mitosis becomes the initial viscosity increase of the second.

7. In Nereis, the viscosity changes between fertilization and cleavage are similar to those found in Cumingia. Apparently these changes are not as marked as in Cumingia, a fact which is correlated with the larger size of the Nereis egg and the relatively small size of its spindle.

8. The discussion includes a critique of the microdissection method of viscosity measurement as well as certain interpretative considerations. 


\section{LITERATURE CITED}

AnnoLd, H. D. 1911 Limitations imposed by slip and inertia terms upon Stokes's law for the motion of spheres through liquids. Phil. Mag., 6th ser., vol. 22 , pp. $755-775$.

ArNDT, K. 1907 Zähigkeitsmessungen bei hohen Temperaturen. Zeitschr. f. Elektrochem., Bd. 13, S. 578-582.

Bayliss, W. M. 1920 The properties of colloidal systems. IV. Reversible gelation in living protoplasm. Proc. Roy. Soc., ser. B, vol. 91, pp. 196-201.

Chambers, R. 1917 a Microdissection studies. The cell aster: a reversible gelation phenomenon. Proc. Amer. Soc. Zoöl., Anat. Rec., vol. 11, p. 491.

$1917 \mathrm{~b}$ Microdissection studies. II. The cell aster: a reversible gelation phenomenon. Jour. Exp. Zoöl., vol. 23, pp. 483-505.

1919 Changes in protoplasmic consistency and their relation to cell division. Jour. Gen. Physiol., vol. 2, pp. 49-68.

Dunstan, A. E., and Thole, F. B. 1914 The viscosity of liquids. London: Longmans Green \& Co.

GRIFFIN, B. B. 1899 Studies on the maturation, fertilization, and cleavage of Thalassema and Zirphaea. Jour. Morph., vol. 15 pp. 583-634.

Hein bronn, A. 1914 Zustand des Plasmas und Reizbarheit. Ein Beitrag zur Physiologie der lebenden Substanz. Jahrb. f. wiss. Bot., Bd. 54, S. $357-390$.

Heilbrunn, L. V. 1915 Studies in artificial parthenogenesis. II. Physical changes in the egg of Arbacia. Biol. Bull., vol. 29, pp. 149-203.

1917 An experimental study of cell division. Proc. Amer. Soc. Zoöl., Anat. Rec., vol. 11, pp. 487-489.

1920 An experimental study of cell division. I. The physical conditions which determine the appearance of the spindle in sea-urchin eggs. Jour. Exp. Zoöl., vol. 30. pp. 211-237.

Jordan, H. E. 1910 A cytological study of the egg of Cumingia, with special reference to the history of the chromosomes and the centrosome. Arch. f. Zellforsch., Bd. 4, S, 243-253.

LADENBURG, R. 1907 Über die innere Reibung zäher Flüssigkeiten und ihre Abhängigkeit von Druck. Annalen der Physik, 4te Folge, Bd. 22, S. 287-309.

Leblond, É. 1919 a Le passage de l'état de gel a l'état de sol dans le protoplasma vivant. C. R. Soc. Biol., T. 82, pp. 1150-1152.

1919 b L'état de sol dans les rapports avec l'activité fonetionnelle du protoplasma. C. R. Soc. Biol., T. 82, pp. 1220-1222.

LiLLIE, F. R. 1901 The organization of the egg of Unio, based on a study of its maturation, fertilization, and cleavage. Jour. Morph., vol. 17, pp. $227-292$.

1912 Studies of fertilization in Nereis. III. The morphology of the normal fertilization of Nereis; IV. The fertilizing power of portions of the spermatozoön. Jour. Exp. Zoöl., vol. 12, pp. 413-476.

Morgan, T. H. 1910 Cytological studies of centrifuged eggs. Jour. Exp. Zoöl., vol. 9, pp. 593-655. 
SEIfRIz, W. 1918 Observations on the structure of protoplasm by aid of microdissection. Biol. Bull., vol. 34, pp. 307-324.

1920 Viscosity values of protoplasm as determined by microdissection. Bot. Gaz., vol. 70, pp. 360-386.

Sтокеs, G. G. 1850 On the effect of the internal friction of fluids on the motion of pendulums. Trans. Cambridge Philos. Soc., vol. 9, pp. [8]-[106], see part I, section IV.

Weber, F. 1918 Die Plasmaviskosität pflanzlicher Zellen. Sammelreferat. Zeitschr. f. allgem. Physiol., Bd. 18, S. 1-20.

Weber, F. AND G. 1917 Die Temperaturabhängigkeit der Plasmaviskosität. Ber. deutsch. bot. Ges., Bd. 34, S. 836-846.

WiLson, E. B. 1892 The cell-lineage of Nereis. A contribution to the cytogeny of the annelid body. Jour. Morph., vol. 6, pp. 361-480. 